\title{
Effects of dielectric gradients-mediated ions partitioning on the electrophoresis of composite soft particles: an analytical theory
}

\author{
Paramita Mahapatra ${ }^{\mathrm{a}}$, Partha P. Gopmandal ${ }^{\mathrm{a}}$, Jérôme F. L. Duval ${ }^{\mathrm{b}}$ \\ a Department of Mathematics, National Institute of Technology Durgapur, Durgapur-713209, India. \\ ${ }^{\mathrm{b}}$ Université de Lorraine, CNRS, Laboratoire Interdisciplinaire des Environnements Continentaux \\ (LIEC), F-54000 Nancy, France.
}

*Corresponding Author. Email: partha.gopmandal@maths.nitdgp.ac.in. Phone: +91-7250276690

\begin{abstract}
In this work, we report original analytical expressions defining the electrophoretic mobility of composite soft particles comprising an inner core and a surrounding polymer shell with differentiated permeabilities to ions from aqueous background electrolyte and to fluid flow developed under applied DC field conditions. The existence of dielectric permittivity gradients operational at the core/shell and shell/solution interfaces is accounted for within the Debye-Hückel approximation and flat plate configuration valid in the thin double layer regime. The proposed electrophoretic mobility expressions, applicable to weakly to moderately charged particles with size well exceeding the Debye layer thickness, involve the relevant parameters describing the particle core/shell structure and the electrohydrodynamic features of the core and shell particle components. It is shown that the analytical expressions reported so far in literature for the mobility of hard (impermeable) or porous particles correspond to asymptotic limits of the more generic results detailed here. The impacts of dielectricmediated effects of ions partitioning between bulk solution and particle body on the electrophoretic response are further discussed. The obtained expressions pave the way for a refined quantitative, analytical interpretation of electrophoretic mobility data collected on soft (nano)particles (e.g. functionalized dendrimers, multilayered polyelectrolytic particles) or biological cells (e.g. viruses) for which the classical hard core-soft shell representation is not appropriate.
\end{abstract}

Keywords: Electrophoresis, Core-shell particles, Soft particles, Ions partitioning effects, Electrostatic softness, Hydrodynamic softness.

\section{Introduction.}

The electrophoretic mobility of colloidal particles is a key parameter from which the density of charges carried by the particles in aqueous solution can be evaluated. In detail, the conversion of particle electrophoretic mobility into zeta-potential via standard electrokinetic models, and the subsequent recourse to Gouy-Chapman electric double layer theory or extensions thereof allow the estimation of 
surface charge density of hard colloids impermeable to ions and fluid flow [1-2]. For the case of soft colloids permeable to ions and fluid flow, e.g. viruses or bacteria [3-6], (bio)functionalized (nano)particles [7-8], recombinant protein particles [9] or environmental humics and fulvics [10,11], the concept of a well-defined zeta-potential (or electrokinetic potential) loses its physical meaning due to the defining hydrodynamic softness of these (nano)particulate systems [5]. The structure of soft particles encompasses generally an inner hard core coated by an ion- and fluid-permeable layer consisting of charged polymeric material [3-11]. The limits of porous and hard colloids correspond to the situations where soft particles lack the inner core and the soft surface layer, respectively.

So far, a large body of literature is available on the modelling of electrophoresis of soft particles with various combinations of density distributions of polymer segments and structural charges across the interphase formed between the bulk particle body and the external electrolyte solution. Assuming an exponential profile for the polymer segments density distribution, Ohshima [12] derived an approximate analytical expression of the electrophoretic mobility of charged polymer-coated particles and Hill et al. [13] investigated numerically the electrophoretic response of particles decorated by brushlike polymer layers. The electrophoresis of polymer coated-particles with linear and exponential distributions of immobile structural charges across the peripheral soft surface layer was further analysed theoretically by Lopez-Garcia et al. [14]. Experimental electrokinetic work on soft microgel particles supported the non-uniform distributions of polymer material from the centre of the particles to the outer aqueous medium in connection to swelling-shrinking processes $[15,16]$. In this context, Duval and Ohshima [17] introduced the concept of soft diffuse interfaces and particles, and modelled how particle electrophoretic mobility is affected by the sigmoidal distributions of polymer segments and of immobile charges density across the soft component of core-shell and porous particles. They analysed numerically the dependence of electrophoretic mobility of such diffuse soft particles over a wide range of relevant electrohydrodynamic parameters including Debye length scale, thickness and extent of the diffuseness of the soft polymer layer. They further successfully confronted theory to experimental data collected on various biological soft particles in particular bacteria and bacteriophages (see [5] and references therein). Last, Duval and co-workers studied how the chemical and structural anisotropies of the polymeric shell determine the dependence of soft particles mobility on electrolyte concentration and solution $\mathrm{pH}$, a feature that is required to analyse the electrophoretic properties of particles defined by layered distribution of anionic and cationic charges and those of viruses [18-20].

Whereas the evaluation of electrokinetics of soft particles with inhomogeneous distributions of polymer segments density across the peripheral shell generally requires numerical modelling, the development of explicit analytical expressions of soft particle mobility is generally possible only for weakly to moderately charged particles with step-like polymer coatings and homogeneous distribution of their structural charges. Within the so-called flat plate representation applicable to particles whose size is sufficiently large compared to the Debye layer thickness, Ohshima and Kondo [21-24] elaborated tractable expressions for the electrophoretic mobility of biological cells assimilated to soft particles with 
step-like repartition of polymer coatings. The dependence of soft particles electrophoretic mobility on particle size was also investigated by Ohshima for various electrostatic scenario [25-29]. Ohshima [30] further studied theoretically the impacts of electric double layer polarization and relaxation that become significant when the magnitude of the particle charge density is sufficiently large. Despite of the necessary approximations made within these analytical theories, the closed-forms expressions derived for the electrophoretic mobility of soft particles have found a wide range of applications for the analysis and interpretation of the electrohydrodynamic features of a large collection of (bio)colloids [4, 6-10, 31-32].

In the aforementioned literature work, the dielectric permittivity of the polymeric layer grafted or sorbed on the particle core is generally considered identical to that of the aqueous background electrolyte medium. Such an assumption is certainly valid for dilute polyelectrolyte systems [33] but it becomes questionable for sufficiently dense polymeric layers where the dielectric permittivity is generally different from that of water. The inner core and the outer shell of composite soft particles are made of polyelectrolytic materials whose dielectric constant may differ from that of bulk electrolyte solution depending on material composition and density [34-37]. For membrane materials, this permittivity is lower than that of water [38]. In turn, the differentiated permittivities of the polymer layer and the solvent may have a significant impact on the electrophoretic properties of soft particles [34-36] or on the electrohydrodynamics of soft nanochannels [37]. Indeed, they generate a difference of Born energies between the polymeric and aqueous phases, which in turn reduces the penetration of mobile ions within the polymeric material or, equivalently, reduces the electrostatic softness of the particles. Such a nonlinear process has been termed 'ions partitioning effect' [39]. Also, particle mobility expressions derived with assuming identical dielectric permittivity between core and shell phases are necessarily approximate in capturing the electrophoretic features of metallic or semiconducting particles functionalized with polymer coatings. Whereas there is an abundant literature on theory of electrophoresis for soft particles with simplified representation of dielectric permittivity distribution throughout the core and shell particle components, lesser attention has been paid to the elaboration of electrokinetic models for core-shell particles with account of dielectric-induced ions partitioning effects $[40,41]$. In addition, the internal particle core has been exclusively tackled with assuming insignificant permeation to ions and flow within it. However, as recently argued by Maurya et al. [42], there are many soft particle types of practical relevance, like functionalized nanodendrimers [19], where the core may also be permeable to ions and/or fluid flow depending on its chemical composition. Accordingly, Maurya et al. [42] introduced the generic term 'composite soft particles' to denote core-shell systems for which not only the shell (hereafter denoted as 'sl') but also the particle core (hereafter denoted as ' $c$ ') may host ions from the background electrolyte and/or be permeable to flow under applied field conditions. This led Maurya et al. [42] to distinguish between different composite soft particles along the lines detailed in Table 1, with the qualification 'soft' (' $\mathrm{S}$ ' in short) and 'semi-soft' ('SS' in short) applying to a particle compartment (core or shell) that is permeable to 
both ions and flow, and to a particle compartment that is permeable to ions but not to flow, respectively. Particles denominated by the abbreviations 'Sc-Ssl', 'SSc-Ssl', 'SSc-SSsl' thus refer to various combinations of flow- and ions-permeability properties in the core and shell regions (Table 1).

\begin{tabular}{|c|c|c|c|c|}
\hline \multirow[b]{2}{*}{$\begin{array}{l}\text { CORE-SHELL } \\
\text { PARTICLE type }\end{array}$} & \multicolumn{2}{|c|}{ CORE } & \multicolumn{2}{|c|}{ SHELL } \\
\hline & $\begin{array}{l}\text { Permeability } \\
\text { to ions }\end{array}$ & $\begin{array}{c}\text { Permeability to } \\
\text { fluid flow }\end{array}$ & $\begin{array}{c}\text { Permeability to } \\
\text { ions }\end{array}$ & $\begin{array}{c}\text { Permeability to } \\
\text { fluid flow }\end{array}$ \\
\hline $\begin{array}{c}\text { Sc-Ssl } \\
\text { Ex.: virus particles [18] }\end{array}$ & YES & YES & YES & YES \\
\hline $\begin{array}{c}\text { SSc-Ssl } \\
\text { Ex.: natural rubber particles } \\
{[43]}\end{array}$ & YES & NO & YES & YES \\
\hline $\begin{array}{c}\text { Hard core-Ssl } \\
\text { Ex.: carboxyl-modified } \\
\text { polymer nanoparticles [44] }\end{array}$ & NO & NO & YES & YES \\
\hline $\begin{array}{c}\text { Hard core-SSsl } \\
\text { Ex.: metallic particles } \\
\text { decorated by a flow- } \\
\text { impermeable polymer } \\
\text { surface layer }\end{array}$ & NO & NO & YES & NO \\
\hline $\begin{array}{c}\text { SSc-SSsl } \\
\text { Ex.: dendrimer nanoparticles } \\
{[19]}\end{array}$ & YES & NO & YES & NO \\
\hline
\end{tabular}

Table 1: Different types of composite semi soft (SS)-soft (S) particles consisting of a polyelectrolyte core (c) decorated by a polyelectrolyte shell layer (sl). The term 'hard' refers to particle compartment that is impermeable to both ions and flow. The mobility expressions derived in this work for Sc-Ssl, SSc-Ssl, SSc-SSsl, hard core-Ssl and hard core-SSsl with account of dielectric-mediated ions partitioning effects are provided by Eqs. (11), (16), (17), (18) and (19), respectively. Examples are provided for each composite particle configuration on the basis of particle electrokinetic analyses reported in the literature.

Maurya et al. [42] derived explicit analytical expressions for the electrophoretic mobility of Sc-Ssl, SSc-Ssl, SSc-SSsl particles within the linearized Poisson-Boltzmann framework valid for low to moderate particle electrostatic potential and they restricted their analysis to the situation of sufficiently dilute polyelectrolytic core and/or shell so that the impacts of dielectric effects could be discarded. In the current study, we extend our findings given in [42] to situations where dielectric-induced ion 
partitioning effects are operational and we develop original electrophoretic mobility expressions applicable to Sc-Ssl, SSc-Ssl, SSc-SSsl, hard core-Ssl and hard core-SSsl particles (Table 1) with explicit account of differences between dielectric permittivities of the electrolyte medium, of the particle core and of the particle shell. It is demonstrated that soft and hard particles mobility expressions previously reported in literature all refer to limits of the more generic expressions elaborated here.

\section{Mathematical formulation.}

In the electrohydrodynamic developments that follow, we adopt the flat-plate configuration applicable to spherical particles with a thin electric double layer compared to the particle radius. The methodology we follow to derive the expressions of particle electrophoretic mobility in the thin double layer limit is illustrated in Ref. 22 by Ohshima and coworkers. The only approximation validating the flat-plate representation for soft particles is that the radius of the particle is much larger than the Debye layer thickness. With this assumption, particles may be treated as a planar core covered by a polyelectrolyte layer of arbitrary thickness and Brinkmann screening length. As such, the expressions derived below are not only applicable to a planar (strictly) surface in contact with an electrolyte (limit where the particle radius goes to infinity) but also to a spherical particle sufficiently large for the thin double layer limit to apply and for the electric field to be parallel to the particle surface. The flat-plate representation was also adopted in other work on soft particles electrophoresis, see e.g. Donath and Pastushenko [45]; and Wunderlich [46]. The planar coordinate system is similar to that given in [42] and, for the sake of convenience, it is recalled in Figure 1.

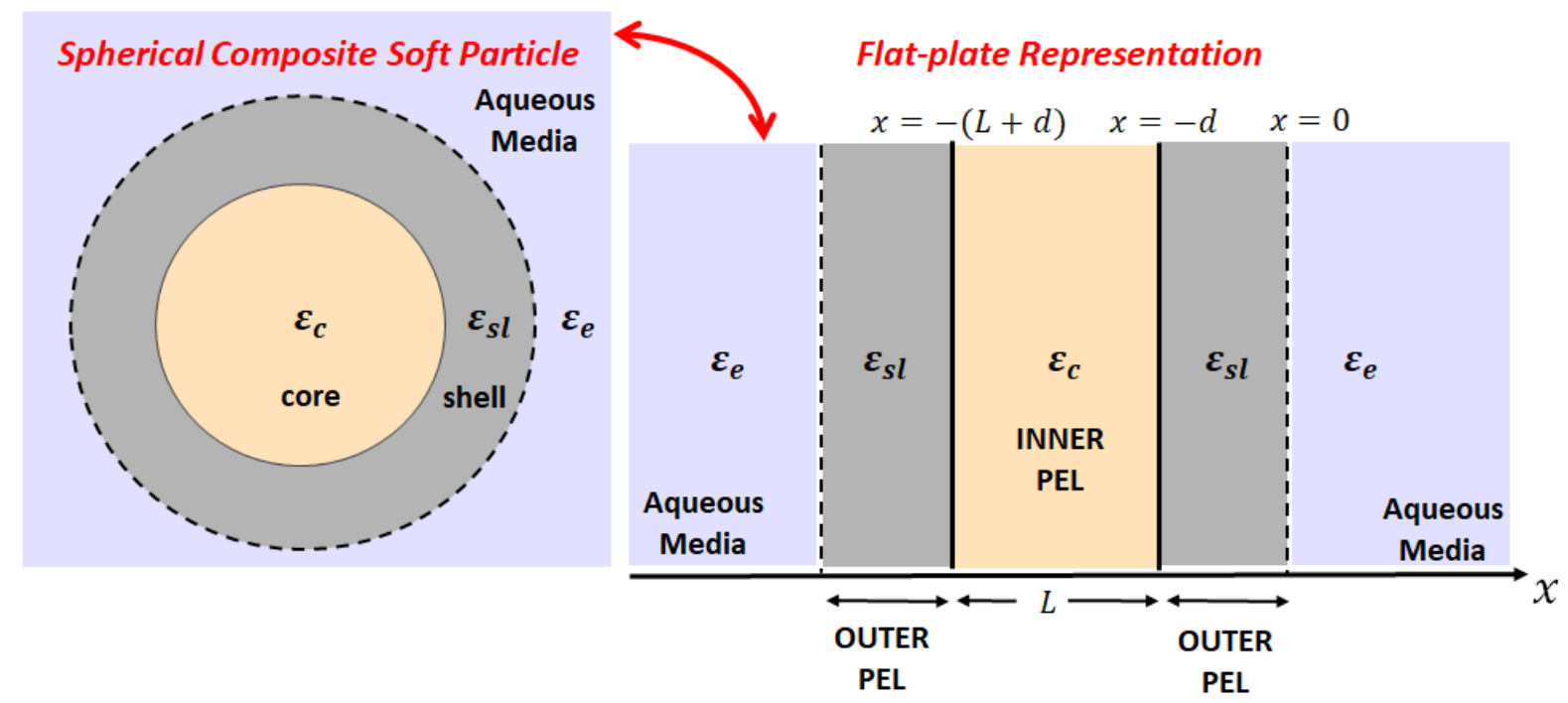

Figure 1. Schematic illustration of a core-shell particle (represented according to the flat-plate configuration, see details in the text) consisting of an inner polyelectrolyte layer (PEL) of thickness $L$ (core component) covered by a peripheral shell component of thickness $d$ (the outer PEL). The dielectric permittivities of the aqueous electrolyte medium and of the particle core and shell components are further specified. 
The shell/electrolyte solution and core/shell interfaces are located at $x=0$ and $x=-d$, respectively. The thickness of the core component is denoted as $L$ and the densities of net amount of structural charges entrapped within the inner core and the outer shell volumes are $\rho_{\text {core }}=z_{c} F N_{c}$ and $\rho_{\text {shell }}=z_{s l} F N_{s l}$, respectively, where $z_{c}, z_{s l}$ and $N_{c}, N_{s l}$ are the valences and concentrations of structural (immobile) charges carried by the core (subscript $c$ ) and by the shell (subscript $s l$ ), respectively. The dielectric permittivities of the electrolyte medium, of the outer shell and of the inner core are hereafter denoted as $\varepsilon_{e}, \varepsilon_{s l}$ and $\varepsilon_{c}$, respectively, with $\varepsilon_{e}>\varepsilon_{s l}$ and $\varepsilon_{e}>\varepsilon_{c}$. Considering the bulk electrolyte as a reference medium, the Born energy [37] required to transfer an ion $i$ from bulk solution to a medium with dielectric permittivity $\varepsilon_{j}$ (with $j=c$, sl) is defined by [35,47]

$$
\Delta W_{i}=\frac{\left(Z_{i} e\right)^{2}}{8 \pi r_{i}}\left[\frac{1}{\varepsilon_{j}}-\frac{1}{\varepsilon_{e}}\right]
$$

where $r_{i}$ is the radius of the $i^{\text {th }}$ ion with valence $Z_{\mathrm{i}}$ and $e$ is the elementary charge. For the sake of simplicity, we consider below a binary $z: z$ electrolyte with the index $i=+,-$ in Eq. (1) running over the cations (+) and anions (-) of the electrolyte. Also, we assume that the radius of the hydrated anions and cations are the same with $r_{\mathrm{i}} \equiv r$ [35]. Accordingly, the partitioning coefficient of mobile electrolyte ions between solution and inner core, and solution and outer shell compartments are defined by

$f_{j}=\exp \left[-\frac{(z e)^{2}}{8 \pi r}\left(\frac{1}{\varepsilon_{j}}-\frac{1}{\varepsilon_{e}}\right) \frac{1}{k_{B} T}\right](j=c, s l)$

where $k_{B}$ and $T$ are the Boltzmann constant and the absolute temperature, respectively. Boltzmann statistics further leads to the spatial distributions of mobile electrolyte ions defined by

$n_{i}(x)=n_{0} \exp \left[-i \frac{z e \psi(x)}{k_{B} T}\right](i=+,-)$

where $n_{i}(i=+,-)$ refers to the number concentration of cations and anions and $n_{0}$ is the bulk number concentration of ions. Due to the difference in Born energies of the electrolyte, core and shell particle compartments, the penetration of mobile electrolyte ions across the inner core compartment and outer shell proceeds in a discontinuous manner. The concentrations of mobile ions $(i=+,-)$ at the locations of the relevant interfaces of the problem $(x=0$ and $=-d)$ follow the relationships

$n_{i}\left(-d^{-}\right)=f_{c} n_{i}\left(-d^{+}\right)$,

$n_{i}\left(0^{-}\right)=f_{s l} n_{i}\left(0^{+}\right)$

For sufficiently low particle charge densities, the electrostatic potential in composite soft particles with ion-penetrable core and ion-penetrable shell (i.e. for the Sc-Ssl, SSc-Ssl, SSc-SSsl particle types, see

Table 1) obeys the linearized Poisson Boltzmann equation according to

$$
\frac{\mathrm{d}^{2} \psi}{\mathrm{d} x^{2}}=\left\{\begin{array}{lr}
\kappa_{c}^{2} \psi-\frac{\rho_{\text {core }}}{\varepsilon_{c}} ; & -\left(\frac{L}{2}+d\right) \leq x<-d \\
\kappa_{\text {sl }}^{2} \psi-\frac{\rho_{\text {shell }} ;}{\varepsilon_{s l}} & -d \leq x<0 \\
\kappa^{2} \psi ; & x \geq 0
\end{array}\right.
$$

where $\kappa^{-1}$ is the classical electric double layer thickness defined by $\kappa^{-1}=\sqrt{\varepsilon_{e} k_{B} T / 2 e^{2} z^{2} n_{0}}$. The quantities $\kappa_{c}$ and $\kappa_{s l}$ are related to $\kappa$ via $\kappa_{c}^{2}=f_{c} \kappa^{2} \varepsilon_{e} / \varepsilon_{c}$ and $\kappa_{s l}^{2}=f_{s l} \kappa^{2} \varepsilon_{e} / \varepsilon_{s l}$, respectively, and 
correspond to the reciprocals of the electric double layer thickness operational in the core and shell region, respectively. We further adopt the continuity conditions for the potential and electric displacement vector along the interfaces at $x=0$ and $x=-d$. In addition, standard symmetry and far field conditions apply for the potential and electric field at $x=-(L / 2+d)$ and in the bulk solution (i.e. $\psi \rightarrow 0$ as $x \rightarrow \infty$ ), respectively. After some algebra, solving Eq. (5) leads to the electrostatic potential distribution from the particle centre to the outer electrolyte defined by

$$
\begin{aligned}
& \psi(x)= \\
& \left\{\begin{array}{lr}
{\left[\psi(-d)-\frac{\rho_{\text {core }}}{\varepsilon_{c} \kappa_{c}^{2}}\right] \operatorname{sech}\left(\frac{\kappa_{c} L}{2}\right) \cosh \left[\kappa_{c}\left(x+\frac{L}{2}+d\right)\right]+\frac{\rho_{\text {core }}}{\varepsilon_{c} \kappa_{c}^{2}} ;} & -\left(\frac{L}{2}+d\right) \leq x<-d \\
\psi_{\text {DON }}\left[1+(P-1) \cosh \left(\kappa_{s l} x\right)-\frac{\varepsilon_{e}}{\varepsilon_{s l}} \frac{\kappa}{\kappa_{s l}} P \sinh \left(\kappa_{s l} x\right)\right] & -d \leq x<0 \\
+Q \frac{\rho_{\text {core }}}{\varepsilon_{e} \kappa_{c}^{2}}\left[\cosh \left(\kappa_{s l} x\right)-\frac{\varepsilon_{e}}{\varepsilon_{s l}} \frac{\kappa}{\kappa_{s l}} \sinh \left(\kappa_{s l} x\right)\right] ; & x \geq 0 \\
\left(\psi_{D O N} P+Q \frac{\rho_{\text {core }}}{\varepsilon_{e} \kappa_{c}^{2}}\right) e^{-\kappa x} ; &
\end{array}\right.
\end{aligned}
$$

where the introduced Donnan potential $\psi_{D O N}$ in the shell and the electrostatic potentials at $x=0$ and $x=-d$ are defined by

$$
\left\{\begin{array}{l}
\psi_{\text {DON }}=\frac{\rho_{\text {shell }}}{\varepsilon_{s l} \kappa_{s l}^{2}} \\
\psi(0)=\psi_{\text {DON }} P+Q \frac{\rho_{\text {core }}}{\varepsilon_{e} \kappa_{c}^{2}} \\
\psi(-d)=\psi_{D O N}\left[1-\cosh \left(\kappa_{s l} d\right)+P\left\{\cosh \left(\kappa_{s l} d\right)+\frac{\varepsilon_{e}}{\varepsilon_{s l}} \frac{\kappa}{\kappa_{s l}} \sinh \left(\kappa_{s l} d\right)\right\}\right] \\
\quad+Q \frac{\rho_{\text {core }}}{\varepsilon_{e} \kappa_{c}^{2}}\left[\cosh \left(\kappa_{s l} d\right)+\frac{\varepsilon_{e}}{\varepsilon_{s l}} \frac{\kappa}{\kappa_{s l}} \sinh \left(\kappa_{s l} d\right)\right]
\end{array}\right.
$$

which further involves the parameters $P$ and $Q$ whose defining expressions are

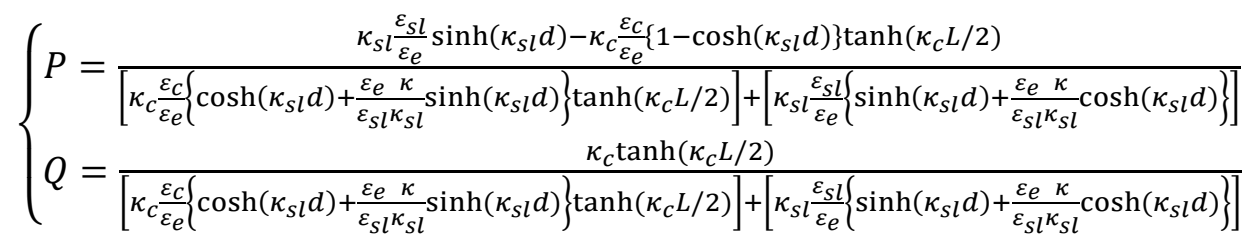

It is recalled that Eqs. (6a)-(6c) apply to Sc-Ssl, SSc-Ssl, SSc-SSsl particle types for which, in particular, the inner core is permeable to ions from the background electrolyte.

Next, we derive the electrostatic potential distribution applicable to composite soft particles with ion-impenetrable inner core (relevant for hard core-Ssl and hard-core SSsl particles types, see Table 1). For such particles, the linearized Poisson-Boltzmann equation governing the electrostatic potential profile is provided by

$$
\frac{\mathrm{d}^{2} \psi}{\mathrm{d} x^{2}}=\left\{\begin{array}{lr}
-\frac{\rho_{\text {core }}}{\varepsilon_{c}} ; & -\left(\frac{L}{2}+d\right) \leq x<-d \\
\kappa_{s l}^{2} \psi-\frac{\rho_{\text {shell }}}{\varepsilon_{s l}} ; & -d \leq x<0 \\
\kappa^{2} \psi ; & x \geq 0
\end{array}\right.
$$


Using the boundaries considered for solving Eq. (5), an explicit expression for the dependence of the electrostatic potential $\psi$ on the position $x$ can be found. After some developments, the result reads as

$$
\psi(x)=\left\{\begin{array}{cr}
\psi_{\text {DON }}\left[1+(R-1) \cosh \left(\kappa_{s l} d\right)+R \frac{\varepsilon_{e}}{\varepsilon_{s l}} \frac{\kappa}{\kappa_{s l}} \sinh \left(\kappa_{s l} d\right)\right]-\frac{\rho_{\text {core }}}{2 \varepsilon_{c}}\left\{\left(x+\frac{L}{2}+d\right)^{2}-\left(\frac{L}{2}\right)^{2}\right\} \\
+\frac{\rho_{\text {core }} L}{2 \varepsilon_{s l} \kappa_{s l}} S\left[\cosh \left(\kappa_{s l} d\right)+\frac{\varepsilon_{e}}{\varepsilon_{s l}} \frac{\kappa}{\kappa_{s l}} \sinh \left(\kappa_{s l} d\right)\right] ; & -\left(\frac{L}{2}+d\right) \leq x<-d \\
\left.\psi_{D O N}\left[1-\cosh \left(\kappa_{s l} x\right)\right]+R\left\{\cosh \left(\kappa_{s l} x\right)-\frac{\varepsilon_{e}}{\varepsilon_{s l}} \frac{\kappa}{\kappa_{s l}} \sinh \left(\kappa_{s l} x\right)\right\}\right] & -d \leq x<0 \\
+\frac{\rho_{\text {core }} L}{2 \varepsilon_{s l} \kappa_{s l}} S\left[\cosh \left(\kappa_{s l} x\right)-\frac{\varepsilon_{e}}{\varepsilon_{s l}} \frac{\kappa}{\kappa_{s l}} \sinh \left(\kappa_{s l} x\right)\right] ; & x \geq 0 \\
{\left[\psi_{\text {DON }} R+\frac{\rho_{\text {core } L} L}{2 \varepsilon_{s l} \kappa_{s l}} S\right] e^{-\kappa x} ;} & -d
\end{array}\right.
$$

where the parameters $R$ and $S$ are defined by

$\left\{\begin{array}{l}R=\frac{\sinh \left(\kappa_{s l} d\right)}{\sinh \left(\kappa_{s l} d\right)+\frac{\varepsilon_{e} \kappa}{\varepsilon_{s l} \kappa_{s l}} \cosh \left(\kappa_{s l} d\right)} \\ S=\frac{1}{\sinh \left(\kappa_{s l} d\right)+\frac{1}{\varepsilon_{S l} \kappa} \kappa_{s l}} \cosh \left(\kappa_{s l} d\right)\end{array}\right.$

In order to derive the flow velocity field distribution, $u(x)$, relevant for the generic Sc-Ssl particles type (all other particle types in Table 1 may be viewed as limiting cases of Sc-Ssl particles for what concerns the hydrodynamic part of the problem, see discussion below), one needs to consider the ion partitioning effects in the formulation of the electromotive force subsumed into the fluid flow equation (i.e. the Darcy-Brinkman equation within the inner core and the peripheral shell, and the Stokes equation in the aqueous medium). Corresponding volumetric body force densities operative in the inner core and in the peripheral shell subjected to an applied electric field of magnitude $E$ are then given by $F_{B, j}=$ $\left(f_{j} e \sum_{i} z_{i} n_{i}\right) E(j=c, s l)$. In addition, in the electrolyte medium the volumetric electric body force density is written $F_{B}=\left(e \sum_{i} z_{i} n_{i}\right) E$. The reader is referred to [42] for a detailed discussion on the governing hydrodynamic equations and associated relevant boundaries. Then, following the strategy adopted in [42], the velocity field $u(x)$ for Sc-Ssl particles with account of ions partitioning effects can be explicitly derived. After some rearrangements, the result can be written in the form

$$
u(x)=\left\{\begin{array}{lr}
C_{1} \cosh \left[\lambda_{c}\left(x+\frac{L}{2}+d\right)\right]+\frac{\varepsilon_{c} E}{\eta}\left[\psi(x)+\lambda_{c} \int_{-\left(\frac{L}{2}+d\right)}^{x}\right. & \left.\sinh \left[\lambda_{c}(x-t)\right] \psi(t) d t\right] \\
-\frac{\rho_{\text {core } E}}{\eta \lambda_{c}^{2}} ; & -\left(\frac{L}{2}+d\right) \leq x<-d \\
C_{2} \cosh \left(\lambda_{s l} x\right)-\frac{\varepsilon_{s l} E \lambda_{s l}}{\eta} \int_{-d}^{0} \cosh \left(\lambda_{s l} t\right) \sinh \left(\lambda_{s l} x\right) \psi(t) d t+\frac{\varepsilon_{s l} E}{\eta} \psi(x) \\
+\frac{\varepsilon_{s l} E \lambda_{s l}}{\eta} \int_{-d}^{x} \sinh \left[\lambda_{s l}(x-t)\right] \psi(t) d t-\frac{\rho_{\text {shell }} E}{\eta \lambda_{s l}^{2}} ; & -d \leq x<0 \\
u(0)-\frac{\varepsilon_{e} E}{\eta}[\psi(0)-\psi(x)] ; & x \geq 0
\end{array}\right.
$$


where $\eta$ is the fluid viscosity. The hydrodynamic (or Brinkman) screening lengths $\lambda_{c}^{-1}$ and $\lambda_{c s l}^{-1}$ in the core and shell particle component, respectively, are given by $\lambda_{c, s l}=\sqrt{\gamma_{c, s l} / \eta}$ where $\gamma_{c}$ and $\gamma_{s l}$ are the coefficients of friction exerted by the inner core and the outer shell on the flow, respectively. The constants $C_{1}$ and $C_{2}$ in Eq. (9a) are further defined by

$$
\begin{aligned}
C_{1}=\frac{\varepsilon_{c} E}{\eta F_{1}} \frac{\lambda_{s l} \sinh \left(\lambda_{s l} \mathrm{~d}\right)}{\sinh \left(\lambda_{c} L / 2\right)} \int_{-\left(\frac{L}{2}+d\right)}^{-d} \sinh \left[\lambda_{c}(d+t)\right] \psi(t) d t \\
\quad-\frac{\varepsilon_{s l} E}{\eta F_{1}} \frac{\lambda_{s l}^{2}}{\lambda_{c}} \frac{\cosh \left(2 \lambda_{s l} d\right)}{\sinh \left(\lambda_{c} L / 2\right)} \int_{-d}^{0} \cosh \left(\lambda_{s l} t\right) \psi(t) d t \\
\quad-\frac{\varepsilon_{c} E}{\eta F_{1}} \frac{\lambda_{c} \cosh \left(\lambda_{s l} d\right)}{\sinh \left(\lambda_{c} L / 2\right)} \int_{-\left(\frac{L}{2}+d\right)}^{-d} \cosh \lambda_{c}(d+t) \psi(t) d t \\
+\frac{\lambda_{s l}}{F_{1} \lambda_{c}} \frac{\sinh \left(\lambda_{s l} d\right)}{\sinh \left(\lambda_{c} L / 2\right)}\left(\frac{\rho_{c o r e} E}{\lambda_{c}^{2} \eta}-\frac{\rho_{s h e l l} E}{\lambda_{s l}^{2} \eta}\right)+\frac{E}{\eta F_{1}} \frac{\lambda_{s l}}{\lambda_{c}} \frac{\sinh \left(\lambda_{s l} d\right)}{\sinh \left(\frac{\lambda_{c} L}{2}\right)}\left(\varepsilon_{s l}-\varepsilon_{c}\right) \psi(-d)
\end{aligned}
$$

$C_{2}=-\frac{\varepsilon_{c} E \lambda_{c}}{\eta F_{1}} \frac{1}{\sinh \left(\frac{\lambda_{c} L}{2}\right)} \int_{-\left(\frac{L}{2}+d\right)}^{-d} \cosh \lambda_{c}\left(\frac{L}{2}+d+t\right) \psi(t) d t-\frac{\varepsilon_{s l} E}{\eta F_{1}}\left\{\frac{\lambda_{s l}^{2}}{\lambda_{c}} \operatorname{coth}\left(\frac{\lambda_{c} L}{2}\right) \cosh \left(\lambda_{s l} d\right)+\right.$

$\left.\lambda_{s l} \sinh \left(\lambda_{s l} d\right)\right\} \int_{-d}^{0} \cosh \left(\lambda_{s l} t\right) \psi(t) d t-\frac{1}{F_{1}}\left(\frac{\rho_{\text {core }} E}{\eta \lambda_{c}^{2}}-\frac{\rho_{\text {shell }} E}{\eta \lambda_{s l}^{2}}\right)-\frac{E}{\eta F_{1}}\left(\varepsilon_{s l}-\varepsilon_{c}\right) \psi(-d)$

Given the flow field defined by Eqs. (9a)-(9c) and further proceeding as detailed in [42], the general expression for the electrophoretic mobility $\mu_{E}$ of Sc-Ssl particles can be derived

$$
\begin{aligned}
& \mu_{E}=\frac{\varepsilon_{c}}{\eta F_{1}}\left[\psi(-d) F_{2}+\frac{\rho_{c o r e}}{\varepsilon_{c} \kappa_{c}^{2}}\left(1-F_{2}\right)\right]+\frac{\varepsilon_{s l}}{\eta F_{1}}\left[\lambda_{s l} \int_{-d}^{0} \sinh \left[\lambda_{s l}(x+d)\right] \psi(x) d x+\right. \\
& \left.\frac{\lambda_{s l}^{2}}{\lambda_{c}} \operatorname{coth}\left(\frac{\lambda_{c} L}{2}\right) \int_{-d}^{0} \cosh \left[\lambda_{s l}(x+d) \psi(x) d x\right]\right]+\frac{\varepsilon_{s l}}{\eta}\left(\frac{\kappa_{s l}}{\lambda_{s l}}\right)^{2} \psi_{D O N}\left\{1-\frac{1}{F_{1}}\right\}+\frac{\rho_{\text {core }}}{\eta \lambda_{c}^{2}} \frac{1}{F_{1}}+ \\
& \frac{1}{\eta F_{1}}\left(\varepsilon_{s l}-\varepsilon_{c}\right) \psi(-d)+\frac{1}{\eta}\left(\varepsilon_{e}-\varepsilon_{s l}\right) \psi(0)
\end{aligned}
$$

where the scalars $F_{1}, F_{2}$ are given by

$$
\left\{\begin{array}{c}
F_{1}=\cosh \left(\lambda_{s l} d\right)+\left(\frac{\lambda_{s l}}{\lambda_{c}}\right) \operatorname{coth}\left(\frac{\lambda_{c} L}{2}\right) \sinh \left(\lambda_{s l} d\right) \\
F_{2}=\frac{1-\left(\frac{\kappa_{c}}{\lambda_{c}}\right) \operatorname{coth}\left(\frac{\lambda_{c} L}{2}\right) \tanh \left(\frac{k_{c} L}{2}\right)}{1-\frac{\kappa_{c}^{2}}{\lambda_{c}^{2}}}
\end{array}\right.
$$

Substituting the potential distribution defined by Eq. (6a) into Eq. (10a), we then derive the desired explicit expression for the electrophoretic mobility of generic Sc-Ssl particles with ions partitioning effects accounted for. After lengthy algebra, the result is recast in the form

$$
\begin{aligned}
& \mu_{E}=\frac{\varepsilon_{e} \psi_{D O N}}{\eta}\left[\frac{\varepsilon_{c} F_{2}}{\varepsilon_{e} F_{1}}\left(\left[1-\cosh \left(\kappa_{s l} d\right)\right]+P\left\{\cosh \left(\kappa_{s l} d\right)+\frac{\varepsilon_{e}}{\varepsilon_{s l}} \frac{\kappa}{\kappa_{s l}} \sinh \left(\kappa_{s l} d\right)\right\}\right)+\frac{\varepsilon_{s l}}{\varepsilon_{e}} \frac{1}{F_{1}}\left(\left[\cosh \left(\lambda_{s l} d\right)-\right.\right.\right. \\
& 1]+(P-1) \frac{\lambda_{s l}^{2}}{\lambda_{s l}^{2}-\kappa_{s l}^{2}}\left\{\cosh \left(\lambda_{s l} d\right)-\cosh \left(\kappa_{s l} d\right)\right\}-\frac{\varepsilon_{e}}{\varepsilon_{s l}} \frac{k}{k_{s l}} P \frac{\lambda_{s l}^{2}}{\lambda_{s l}^{2}-\kappa_{s l}^{2}}\left\{\sinh \left(k_{s l} d\right)-\frac{k_{s l}}{\lambda_{s l}} \sinh \left(\lambda_{s l} d\right)\right\}+ \\
& \frac{\lambda_{s l}}{\lambda_{c}} \operatorname{coth}\left(\frac{\lambda_{c} L}{2}\right)\left\{\sinh \left(\lambda_{s l} d\right)+(P-1) \frac{\lambda_{s l}^{2}}{\lambda_{s l}^{2}-\kappa_{s l}^{2}}\left(\sinh \left(\lambda_{s l} d\right)-\frac{\kappa_{s l}}{\lambda_{s l}} \sinh \left(\kappa_{s l} d\right)\right)-\right.
\end{aligned}
$$




$$
\begin{aligned}
& \left.\left.\frac{\varepsilon_{e}}{\varepsilon_{s l}} \frac{\kappa \lambda_{s l}}{\lambda_{s l}^{2}-\kappa_{s l}^{2}} P\left(\cosh \left(\kappa_{s l} d\right)-\cosh \left(\lambda_{s l} d\right)\right)\right\}\right)+\frac{\varepsilon_{s l}}{\varepsilon_{e}}\left(\frac{\kappa_{s} l}{\lambda_{s l}}\right)^{2}\left(1-\frac{1}{F_{1}}\right)+\left(\frac{\varepsilon_{s} l}{\varepsilon_{e}}-\frac{\varepsilon_{c}}{\varepsilon_{e}}\right) \frac{1}{F_{1}}([1- \\
& \left.\left.\left.\cosh \left(\kappa_{s l} d\right)\right]+P\left\{\cosh \left(\kappa_{s l} d\right)+\frac{\varepsilon_{e}}{\varepsilon_{s l}} \frac{\kappa}{\kappa_{s l}} \sinh \left(\kappa_{s l} d\right)\right\}\right)+P\left(1-\frac{\varepsilon_{s l}}{\varepsilon_{e}}\right)\right]+\frac{\rho_{c o r e}}{\eta \kappa_{c}^{2}}\left[\frac { \varepsilon _ { c } } { \varepsilon _ { e } } \frac { F _ { 2 } } { F _ { 1 } } Q \left(\cosh \left(\kappa_{s l} d\right)+\right.\right. \\
& \left.\frac{\varepsilon_{e}}{\varepsilon_{s l}} \frac{\kappa}{\kappa_{s l}} \sinh \left(\kappa_{s l} d\right)\right)+\left(\frac{1-F_{2}}{F_{1}}\right)+\frac{\varepsilon_{s l} l}{\varepsilon_{e}} \frac{1}{F_{1}} Q\left\{\frac{\lambda_{s l}^{2}}{\lambda_{s l}^{2}-\kappa_{s l}^{2}}\left\{\cosh \left(\lambda_{s l} d\right)-\cosh \left(\kappa_{s l} d\right)\right\}-\right. \\
& \frac{\varepsilon_{e}}{\varepsilon_{s l}} \frac{k}{k_{s l}} \frac{\lambda_{s l}^{2}}{\lambda_{s l}^{2}-\kappa_{s l}^{2}}\left(\sinh \left(k_{s l} d\right)-\frac{k_{s l}}{\lambda_{s l}} \sinh \left(\lambda_{s l} d\right)\right)+\frac{\lambda_{s l}}{\lambda_{c}} \operatorname{coth}\left(\frac{\lambda_{c} L}{2}\right)\left(\frac{\lambda_{s l}^{2}}{\lambda_{s l}^{2}-\kappa_{s l}^{2}}\left(\sinh \left(\lambda_{s l} d\right)-\frac{\kappa_{s l}}{\lambda_{s l}} \sinh \left(\kappa_{s l} d\right)\right)-\right. \\
& \left.\left.\frac{\varepsilon_{e}}{\varepsilon_{s l}} \frac{\kappa \lambda_{s l}}{\lambda_{s l}^{2}-\kappa_{s l}^{2}}\left(\cosh \left(\kappa_{s l} d\right)-\cosh \left(\lambda_{s l} d\right)\right)\right)\right\}+\frac{\kappa_{c}^{2}}{\lambda_{c}^{2}} \frac{1}{F_{1}}+\left(\frac{\varepsilon_{s l}}{\varepsilon_{e}}-\frac{\varepsilon_{c}}{\varepsilon_{e}}\right) \frac{1}{F_{1}} Q\left(\cosh \left(\kappa_{s l} d\right)+\frac{\varepsilon_{e}}{\varepsilon_{s l}} \frac{\kappa}{\kappa_{s l}} \sinh \left(\kappa_{s l} d\right)\right)+ \\
& \left.Q\left(1-\frac{\varepsilon_{s l}}{\varepsilon_{e}}\right)\right]
\end{aligned}
$$

Eq. (11) is one of the important findings of this work. It involves explicitly the parameters defining the particle size and the dimensions of the core and shell components, the defining electrostatic and hydrodynamic properties of the particle as well as the parameters featuring the dielectric-mediated reduction of ion penetration in the core and shell.

Below, we report tractable closed-form expressions of the electrophoretic mobility of Sc-Ssl particle type under various limiting cases. At sufficiently large electrolyte concentration where the inequalities $\kappa>>1, \kappa_{c}>>1$ and $\kappa_{s l}>>1$ apply, the electrophoretic mobility expression (11) reduces to

$\mu_{E}=\frac{\rho_{\text {shell }}}{\eta \lambda_{s l}^{2}}\left(1-\frac{1}{F_{1}}\right)+\frac{\rho_{\text {core }}}{\eta \lambda_{c}^{2}} \frac{1}{F_{1}}$

which corresponds to the non-zero mobility plateau value reached at large electrolyte concentrations where electrostatics is completely screened, a property that is specific to soft particles.

Next we consider the situation of large Sc-Ssl particles in line with the conditions $\lambda_{c} L \gg 1, \lambda_{s l} d \gg$ $1, \kappa_{c} L \gg 1, \kappa_{s l} d \gg 1$, generally applicable -in first order approximation- to biological particles like bacteria [4,5,21-26]. Under such conditions, Eq. (11) simplifies into

$\mu_{E}=\frac{\varepsilon_{s l}}{\eta}\left(\frac{\frac{\psi_{D O N}}{\lambda_{s l}}+\frac{\psi(0)}{\kappa_{s l}}}{\frac{1}{\kappa_{s l}}+\frac{1}{\lambda_{s l}}}\right)+\frac{\rho_{s h e l l}}{\eta \lambda_{s l}^{2}}+\frac{1}{\eta}\left(\varepsilon_{e}-\varepsilon_{s l}\right) \psi(0)$

where $\psi(0)=\psi_{D O N} /\left(1+\frac{\varepsilon_{e}}{\varepsilon_{s l}} \frac{\kappa}{\kappa_{s l}}\right)$ is the electrostatic potential at the surface of the peripheral shell. In the limit $\varepsilon_{s l}=\varepsilon_{e}$, Eq. (13) further reduces to the famous result by Ohshima [26] (Eq. (11.4.18) therein) derived with ignoring dielectric-induced ions partitioning effects. Eq. (13) can be used for fitting electrophoretic mobility data measured on sufficiently large particles (and/or at sufficiently large electrolyte concentrations) as a function of electrolyte concentration upon adjustment of the outer shell charge density, the hydrodynamic softness of the soft surface layer and the ratio $\varepsilon_{s l} / \varepsilon_{e}$ between outer shell and solution permittivities. The adjustment of these three parameters can be performed using e.g. Levenberg-Marquardt algorithm as adopted in [48]. In the case where experimentalists have at their disposal independent data that allow estimation of the ratio $\varepsilon_{s l} / \varepsilon_{e}$ (e.g. via dielectric relaxation spectroscopy measurements), this quantity is no longer an unknown of the problem and the parameters to be adjusted for recovering the dependence of particle mobility on electrolyte concentration are then 
the only charge density and hydrodynamic softness of the surface layer, as classically done on the basis of Ohshima's expression [48], albeit with proper account of the way the electrostatic potential distribution is affected by the dielectric permittivities of the core and shell particle regions.

Mobility expression (13) further corresponds to the well-known Smoluchowski equation [49] in the extreme where shell layer is impermeable to flow $\left(\lambda_{s l} \rightarrow \infty\right)$, i.e.

$\mu_{E}=\frac{\varepsilon_{e}}{\eta} \psi(0)$

, which corresponds to the electrophoretic mobility of a hard colloid with surface potential $\psi_{D O N} / 2$.

With the above results for Sc-Ssl particles at hand, we now provide the mobility expressions relevant for SSc-Ssl, SSc-SSsl, hard core-Ssl, hard core-SSsl particle types (Table 1). For that purpose, we need to implement the condition $\lambda_{c}^{-1} \rightarrow 0$ into the generic mobility expression (10). Accordingly, after developments, the general form of the electrophoretic mobility of composite soft particle with inner core impermeable to fluid flow (relevant for SSc-Ssl, SSc-SSsl, hard core-Ssl, hard core-SSsl particles) is provided by

$$
\begin{aligned}
& \mu_{E}=\frac{\varepsilon_{c}}{\eta \cosh \left(\lambda_{s l} d\right)}[\psi(-d)]+\frac{\varepsilon_{s l}}{\eta \cosh \left(\lambda_{s l} d\right)} \lambda_{s l} \int_{-d}^{0} \sinh \left[\lambda_{s l}(d+t)\right] \psi(t) d t+\frac{\varepsilon_{s l}}{\eta}\left(\frac{\kappa_{s l}}{\lambda_{s l}}\right)^{2} \psi_{D O N}\{1- \\
& \left.\frac{1}{\cosh \left(\lambda_{s l} d\right)}\right\}+\frac{1}{\eta \cosh \left(\lambda_{s l} d\right)}\left(\varepsilon_{s l}-\varepsilon_{c}\right) \psi(-d)+\frac{1}{\eta}\left(\varepsilon_{e}-\varepsilon_{s l}\right) \psi(0)
\end{aligned}
$$

Further using the electrostatic potential distribution given by Eq. (6a), the explicit expression of the electrophoretic mobility of SSc-Ssl particles reads as

$$
\begin{aligned}
& \mu_{E}=\frac{\varepsilon_{s l}}{\eta} \psi_{D O N}\left[\frac{\kappa_{s l}^{2}}{\kappa_{s l}^{2}-\lambda_{s l}^{2}}\left(1-\frac{\cosh \left(\kappa_{s l} d\right)}{\cosh \left(\lambda_{s l} d\right)}\right)+P \frac{\kappa_{s l}^{2}}{\kappa_{s l}^{2}-\lambda_{s l}^{2}} \frac{1}{\cosh \left(\lambda_{s l} d\right)}\left\{\cosh \left(\kappa_{s l} d\right)+\frac{\varepsilon_{e}}{\varepsilon_{s l}} \frac{\kappa}{\kappa_{s l}} \sinh \left(\kappa_{s l} d\right)\right\}+\right. \\
& \left.P \frac{\lambda_{s l}^{2}}{\lambda_{s l}^{2}-\kappa_{s l}^{2}}\left\{1+\frac{\varepsilon_{e}}{\varepsilon_{s l}} \frac{\kappa}{\lambda_{s l}} \tanh \left(\lambda_{s l} d\right)\right\}+\left(\frac{\kappa_{s l}}{\lambda_{s l}}\right)^{2}\left\{1-\frac{1}{\cosh \left(\lambda_{s l} d\right)}\right\}+\left(\frac{\varepsilon_{e}}{\varepsilon_{s l}}-1\right) P\right]+ \\
& \frac{\varepsilon_{s l}}{\varepsilon_{e}} \frac{\rho_{c o r e}}{\eta \kappa_{c}^{2}} Q\left[\frac{\kappa_{s l}^{2}}{\kappa_{s l}^{2}-\lambda_{s l}^{2}} \frac{1}{\cosh \left(\lambda_{s l} d\right)}\left\{\cosh \left(\kappa_{s l} d\right)+\frac{\varepsilon_{e}}{\varepsilon_{s l}} \frac{\kappa}{\kappa_{s l}} \sinh \left(\kappa_{s l} d\right)\right\}+\frac{\lambda_{s l}^{2}}{\lambda_{s l}^{2}-\kappa_{s l}^{2}}\left\{1+\frac{\varepsilon_{e}}{\varepsilon_{s l}} \frac{\kappa}{\lambda_{s l}} \tanh \left(\lambda_{s l} d\right)\right\}+\right. \\
& \left.\left(\frac{\varepsilon_{e}}{\varepsilon_{s l}}-1\right)\right]
\end{aligned}
$$

which reduces, in the limit $\lambda_{s l}^{-1} \rightarrow 0$, to the mobility expression applicable to SSc-SSsl particles type

$$
\mu_{E}=\frac{\varepsilon_{e}}{\eta}\left(\psi_{D O N} P+\frac{\rho_{\text {core }}}{\varepsilon_{e} \kappa_{c}^{2}} Q\right)
$$

As far as the electrophoretic mobility of hard core-Ssl particles is concerned, its expression is easily derived after substitution of the electrostatic potential given by Eq. (8) into Eq. (15), with the result

$$
\begin{aligned}
& \mu_{E}=\frac{\varepsilon_{s l}}{\eta} \psi_{D O N}\left[\frac{\kappa_{s l}^{2}}{\kappa_{s l}^{2}-\lambda_{s l}^{2}}\left(1-\frac{\cosh \left(\kappa_{s l} d\right)}{\cosh \left(\lambda_{s l} d\right)}\right)+R \frac{\lambda_{s l}^{2}}{\lambda_{s l}^{2}-\kappa_{s l}^{2}}\left\{1+\frac{\varepsilon_{e}}{\varepsilon_{s l}} \frac{\kappa}{\lambda_{s l}} \tanh \left(\lambda_{s l} d\right)\right\}+\right. \\
& \left.R \frac{\kappa_{s l}^{2}}{\kappa_{s l}^{2}-\lambda_{s l}^{2}} \frac{1}{\cosh \left(\lambda_{s l} d\right)}\left\{\cosh \left(\kappa_{s l} d\right)+\frac{\varepsilon_{e}}{\varepsilon_{s l}} \frac{\kappa}{\kappa_{s l}} \sinh \left(\kappa_{s l} d\right)\right\}+\left(\frac{\kappa_{s l}}{\lambda_{s l}}\right)^{2}\left(1-\frac{1}{\cosh \left(\lambda_{s l} d\right)}\right)+\left(\frac{\varepsilon_{e}}{\varepsilon_{s l}}-1\right) R\right]+
\end{aligned}
$$




$$
\begin{aligned}
& \frac{\rho_{c o r e} L}{2 \eta \kappa_{s l}}\left[S \frac{\kappa_{s l}^{2}}{\kappa_{s l}^{2}-\lambda_{s l}^{2}} \frac{1}{\cosh \left(\lambda_{s l} d\right)}\left(\cosh \left(\kappa_{s l} d\right)+\frac{\varepsilon_{e}}{\varepsilon_{s l}} \frac{\kappa}{\kappa_{s l}} \sinh \left(\kappa_{s l} d\right)\right)+S \frac{\lambda_{s l}^{2}}{\lambda_{s l}^{2}-\kappa_{s l}^{2}}\left(1+\frac{\varepsilon_{e}}{\varepsilon_{s l}} \frac{\kappa}{\lambda_{s l}} \tanh \left(\lambda_{s l} d\right)\right)+\right. \\
& \left.\left(\frac{\varepsilon_{e}}{\varepsilon_{s l}}-1\right) S\right]
\end{aligned}
$$

Taking the limit $\lambda_{s l}^{-1} \rightarrow 0$ describing particles where the shell is impermeable to flow, Eq. (18) reduces to the mobility expression applicable for hard core-SSsl particles, i.e.

$\mu_{E}=\psi_{D O N} \frac{\varepsilon_{e}}{\eta} R+\frac{\rho_{c o r e} L}{2 \eta \kappa_{s l}} \frac{\varepsilon_{e}}{\varepsilon_{s l}} S$

It was systematically verified that the mobility expressions (11), (16), (17), (18) and (19) relevant for Sc-Ssl, SSc-Ssl, SSc-SSsl, hard core-Ssl and hard core-SSsl particle types, respectively, all merge correctly with the expressions derived by Maurya et al. [42] in the limits $\varepsilon_{c}=\varepsilon_{s l}=\varepsilon_{e}$ where there are no dielectric-mediated ions partitioning effects. The FORTRAN program we developed for electrophoretic mobility computations on the basis of Eqs. (11), (16), (17), (18) and (19) is available on demand.

\section{Computational Illustrations.}

In this section, on the basis of selected computations we briefly illustrate how ions partitioning effects impact on the dimensionless electrophoretic mobility $\mu_{E} / \mu_{0}$ (with $\mu_{0}=\varepsilon_{e} R T /(\eta F)$ ) of the particle types of interest in this work (Table 1). The dielectric permittivity of the background electrolyte medium is set to $\varepsilon_{e}=80 \varepsilon_{0}$ with $\varepsilon_{0}$ the dielectric permittivity of vacuum. In the following developments, the electrostatic softness of the shell and core components is defined by the shell-toelectrolyte medium permittivity ratio $\left(\varepsilon_{s l} / \varepsilon_{e}\right)$ and by the core-to-shell permittivity ratio $\left(\varepsilon_{c} / \varepsilon_{s l}\right)$, respectively. Generally, values of $\varepsilon_{c} / \varepsilon_{s l}$ and $\varepsilon_{s l} / \varepsilon_{e}$ are in the range 0 to 1 depending on the respective chemical compositions of the core and shell particle domains. When dielectric permittivities of the core and shell are identical to that of the electrolyte medium, i.e. $\varepsilon_{c}=\varepsilon_{s l}=\varepsilon_{e}$, the partitioning of mobile ions proceeds in a continuous manner. Such a situation refers to the situation where dielectric-mediated ions partitioning plays no role and we denote this reference situation as that where electrostatic softness of both core and peripheral shell is maximum. For cases where $0<\varepsilon_{s l} / \varepsilon_{e}<1$, decreasing values of $\varepsilon_{s l} / \varepsilon_{e}$ generates a reduction of the transfer of mobile electrolyte ions from the bulk solution to the peripheral particle shell or, equivalently, a decrease in the electrostatic softness of the shell. Similar arguments hold for the electrostatic softness of the core compartment that decreases with decreasing $\varepsilon_{c} / \varepsilon_{s l}$ at fixed $\varepsilon_{s l}$ as a result of reduced ions transfer from the shell to the inner core. 


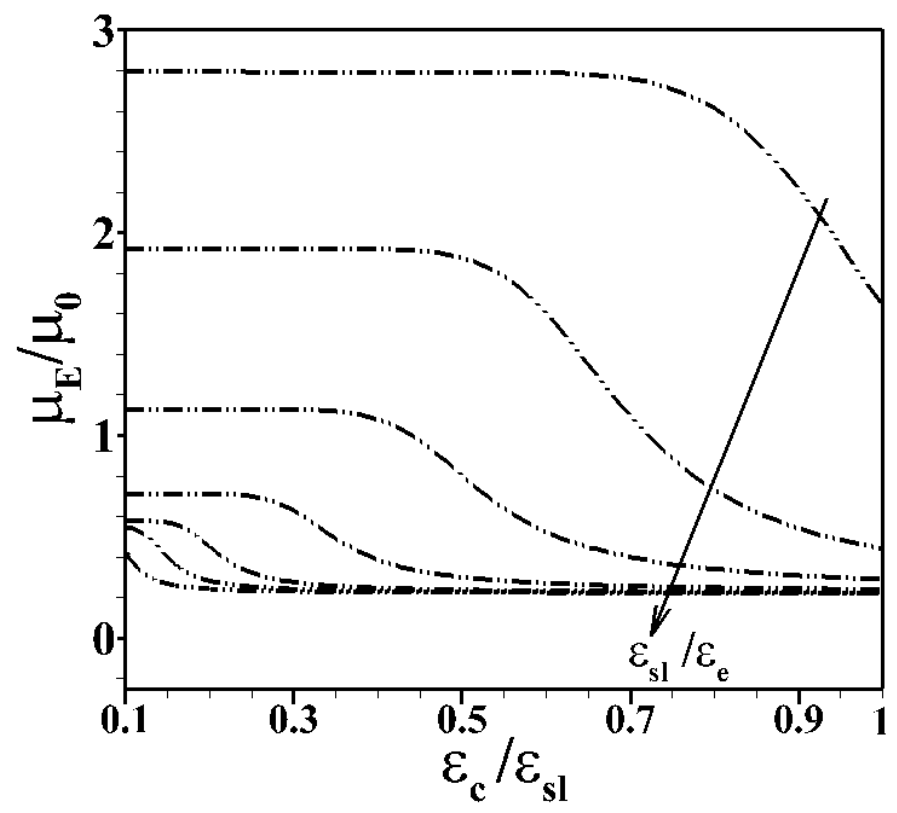

Figure 2. Dependence of scaled mobility $\left(\mu_{E} / \mu_{0}\right)$ of Sc-Ssl particles (Eq. 11) on the electrostatic softness of the core $\left(\varepsilon_{c} / \varepsilon_{s l}\right)$ for various values of the shell electrostatic softness $\varepsilon_{s l} / \varepsilon_{e}=0.1,0.15,0.2$, $0.3,0.5,0.7,1$ at fixed $\lambda_{s l}^{-1}=10 \mathrm{~nm}$ and $\lambda_{c}^{-1}=1 \mathrm{~nm}$. Other model parameters: $N_{c}=N_{s l}=1 \mathrm{mM}, z_{c}=$ $z_{s l}=1, \kappa^{-1}=1 \mathrm{~nm}, d=10 \mathrm{~nm}, L=100 \mathrm{~nm}$. The direction of the arrow represents increasing values of $\varepsilon_{s l} / \varepsilon_{e}$.

Fig. 2 reports the basic dependence of the dimensionless electrophoretic mobility $\mu_{E} / \mu_{0}$ of Sc-Ssl particles (Eq. 11) on the electrostatic softness of the inner core $\left(\varepsilon_{c} / \varepsilon_{s l}\right)$ and that of the peripheral surface layer $\left(\varepsilon_{s l} / \varepsilon_{e}\right)$. Under the here examined conditions, at fixed shell (or core) permittivity, $\mu_{E} / \mu_{0}$ decreases with increasing the permittivity of the inner core (or that of the shell, respectively). Increasing values of $\varepsilon_{c} / \varepsilon_{s l}$ and $\varepsilon_{s l} / \varepsilon_{e}$ at fixed $\varepsilon_{s l}$ and $\varepsilon_{c}$, respectively, lead to an increase of the transferred mobile ions to the core and shell particle regions, which is accompanied by an enhanced accumulation of counterions therein, a subsequent increase of the screening of the electrostatic potential (not shown) and, in turn, a reduction of the particle electrophoretic mobility. It is observed that at sufficiently low ratio $\varepsilon_{c} / \varepsilon_{s l}, \mu_{E} / \mu_{0}$ becomes solely dependent on the dielectric features of the particle shell layer. This asymptotic regime effectively corresponds to that where the exclusion of ions from the core becomes so important that the Sc-Ssl particles basically assimilates to a hard core-Ssl particle from an electrokinetic point of view. In line with this, we systematically verified that at sufficiently low $\varepsilon_{c} / \varepsilon_{s l}$ the electrostatic potentials operational at the core/shell and shell/solution interfaces of Sc-Ssl particles are identical to those computed in the hard core-Ssl particle configuration. Also, upon decreasing the ratio $\varepsilon_{s l} / \varepsilon_{e}$ the mobility plateau reached at low $\varepsilon_{c} / \varepsilon_{s l}$ (which corresponds to the mobility of hard coreSsl particle type, see above discussion) is reached over a larger range of $\varepsilon_{c} / \varepsilon_{s l}$ values. Here, the Sc-Ssl particle behaves effectively as a hard core-Ssl particle with reduced ions accumulation in the shell compartment. 
The most appropriate particle candidate to illustrate the merit of our theory would be metallic particles covered by a polyelectrolyte layer. As already shown in literature for various core-shell particle types, confrontation between experiments and theory requires a refined measurement of particle electrophoretic mobility over a large range of electrolyte concentrations (typically 1-100 mM) and pH (see e.g. [10], [19], [43], [44]). Unfortunately, the literature lacks such exhaustive data measurements for polyelectrolyte-covered metallic particles whose electrokinetic monitoring is often restricted to a (single) measurement point (and conversion thereof into zeta potential) collected at a given $\mathrm{pH}$ and medium salinity. Despite of such a difficulty, the merit of our theory in improving existing classical soft particle electrokinetic predictions can be already inferred qualitatively from Fig. 2. Indeed, the latter indicates a lowering of particle mobility (in absolute value) with decreasing dielectric permittivity in the peripheral particle region that is generally the most active particulate component from an electrokinetic point of view (see [5]). Recalling that particle mobility predicted by Ohshima's theory (eq 13 taken in the limit the limit $\varepsilon_{\mathrm{sl}}=\varepsilon_{\mathrm{e}}$ ) systematically overestimates the magnitude of mobility measurement at sufficiently low electrolyte concentrations (see e.g. [50]), our theory possibly offers a way to counterbalance the discrepancy between experimental data and classical soft electrokinetic theory predictions that ignore the partitioning of ions as mediated by dielectric gradients.

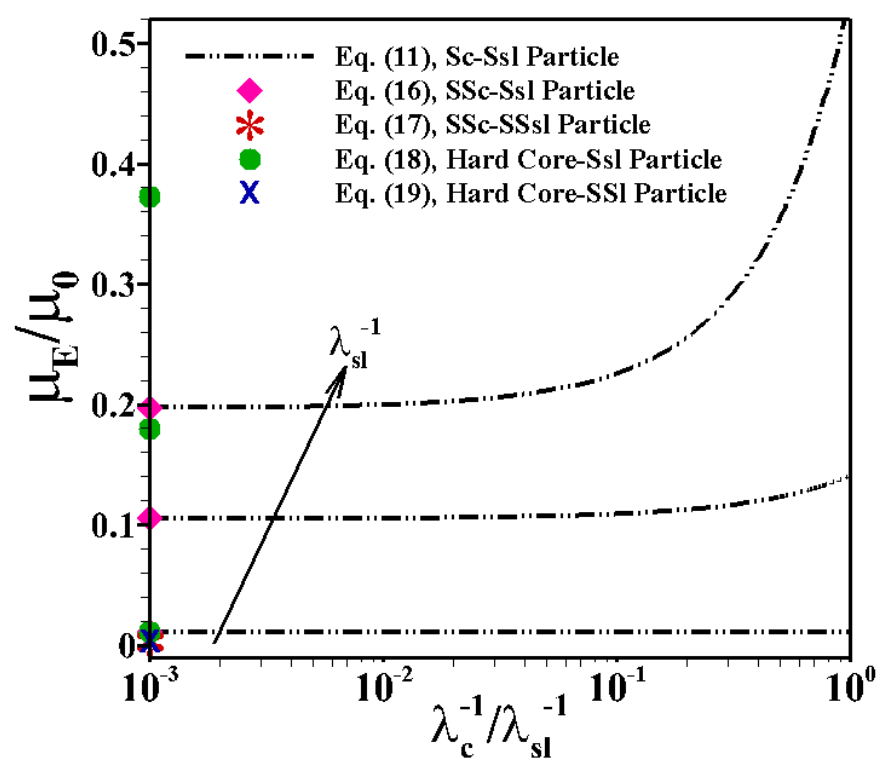

Figure 3. Dependence of scaled electrophoretic mobility $\left(\mu_{E} / \mu_{0}\right)$ of Sc-Ssl particles on the ratio $\lambda_{c}^{-1} / \lambda_{s l}^{-1}$ for various values of $\lambda_{s l}^{-1}(=1,5$ and $10 \mathrm{~nm})$ at fixed $\varepsilon_{c}=0.5 \varepsilon_{e}$ and $\varepsilon_{s l}=0.75 \varepsilon_{e}$. Other model parameters: $N_{c}=N_{s l}=1 \mathrm{mM}, z_{c}=z_{s l}=1, \kappa^{-1}=1 \mathrm{~nm}, d=10 \mathrm{~nm}, L=100 \mathrm{~nm}$. The direction of the arrow represents increasing values of $\lambda_{s l}^{-1}$. Symbols correspond to predictions from Eqs. (16)-(19) for the particle types (mentioned in the figure) where there is no flow penetration in the core component (i.e. $\lambda_{c}^{-1} / \lambda_{s l}^{-1} \rightarrow 0$ ). Predictions from Eqs. (16) and (18) are provided for the three tested values of $\lambda_{s l}^{-1}$ (increasing values of $\lambda_{s l}^{-1}$ from bottom to top). Such computations with Eqs. (17) and (19) lead to results that are independent of $\lambda_{s l}^{-1}$ as the concerned particles then integrate a shell that is impermeable to fluid flow). 
Fig. 3 illustrates the effects of the hydrodynamic softness of the core and shell regions on the electrophoretic mobility of Sc-Ssl particles. Results are reported as a function of the dimensionless ratio $\lambda_{c}^{-1} / \lambda_{s l}^{-1}$ for different values of $\lambda_{s l}^{-1}$, all other relevant model parameters being fixed. For the sake of comparison, the scaled mobility of SSc-Ssl (Eq. 16), SSc-SSsl (Eq. 17), hard core-Ssl (Eq. 18) and hard core-SSsl (Eq. 19) are also reported: they all correspond to situation where there is no flow penetration in the particle core region, i.e. $\lambda_{c}^{-1} \rightarrow 0$. As expected, the mobility $\mu_{E} / \mu_{0}$ of Sc-Ssl particles increases with increasing the hydrodynamic penetration length scales in the core and shell layer $\left(\lambda_{c}^{-1}\right.$ and $\lambda_{s l}^{-1}$, respectively), in agreement with previous conclusions [42]. This trend stems from the associated reduction of the frictions exerted by the core and shell components on the flow and from the resulting increase in electroosmotic flow. At sufficiently low $\lambda_{c}^{-1}$, the scaled mobility $\mu_{E} / \mu_{0}$ of Sc-Ssl particles tends correctly to the plateau value given by Eq. (16) applicable to SSc-Ssl particles defined by the absence of flow in the inner core. At sufficiently low values of both $\lambda_{s l}^{-1}$ and $\lambda_{c}^{-1}$, the electrophoretic mobility of Sc-Ssl particles further satisfactorily compares with that of SSc-SSsl particles (Eq. 17). Under the conditions of Fig. 3, the scaled electrophoretic mobility defined by Eq. (18) relevant for hard core-Ssl particles is systematically larger than that of Sc-Ssl particles (Eq. 11), except for the case $\lambda_{c}^{-1} / \lambda_{s l}^{-1} \rightarrow 0$ where they are identical. This finding is explained by differentiated dielectric-mediated ions partitioning effects operational at the very hard core surface for hard core-Ssl particles, with a resulting shoot of the hard core surface potential, or operational within the full core volume of Sc-Ssl particles with a resulting lower potential at the core/shell interface and thus a lower electrophoretic mobility for the latter particle type. Finally, the mobility of hard core-Ssl particles reduces consistently to that of hard core-SSsl particles (Eq. 19) at sufficiently low $\lambda_{s l}^{-1}$. The conclusions derived from Figures 2-3 may change for highly charged layers for which numerical theories are necessarily required to solve the complete set of electrohydrodynamic equations beyond the linearized Poisson-Boltzmann framework adopted here (this framework is valid for weakly to moderately charged particles). For highly charged layers, relaxation/polarization of electric double layers and related retardation effects on electrophoresis may come into play.

\section{Conclusions.}

There is an abundant literature on experimental and theoretical reports demonstrating that electrophoretic behaviour of soft particles significantly differs from that of the historical case of hard impermeable colloids. Following the original definition by Ohshima [26], soft particles generally refer to (i) core-shell particles where the core, unlike the shell component, is strictly impermeable to ions and to electroosmotic flow, and (ii) porous particles devoid of intra-particulate impermeable core region. This classification excludes however core-shell particles of practical relevance (e.g. dendrimers, viruses or multilayered particles to quote only a few) defined by a polyelectrolyte-like shell supported by a polyelectrolytic core in the volume of which structural charges are distributed. Depending on the nature 
and density of the materials composing such particles, the core and shell volumes may be accessible to ions from background electrolyte and/or to fluid flow, and they may further differ in terms of dielectric properties. Here, we report an analytical theory for the electrophoresis of such a generic type of coreshell particles defined by various combinations of hydrodynamic and electrostatic properties pertaining to their core and shell components and with full account of dielectric-induced ions partitioning effects. We show that the original mobility expressions elaborated in this work are fully consistent with those corresponding to simpler particle structures considered in previous studies. Within the approximations underlying the validity of our analytical formalism, the results further allow the identification of the respective contributions of the core and particle shell domains in determining the electrophoretic mobility of the particle. They also highlight how gradients of dielectric permittivity at the core/shell/solution interfaces are involved in the defining distributions of the electrostatic potential and electroosmotic flow field and, in turn, in the expressions of the particle electrophoretic mobility. Results are further discussed with help of computational examples given for various sets of screening hydrodynamic length scales and electrostatic softness of the core and shell particle compartments. These results are a useful starting point for the evaluation of the electrophoretic properties of complex colloids with complex (bio)polymer-based surface/volume functionalizations as employed in e.g. contaminants remediation applications in aquatic environmental media or nanoparticle-based therapeutics.

Acknowledgements $\underline{P}$. Mahapatra wishes to acknowledge the Council of Scientific and Industrial Research, Govt. of India for financial support in the form of a Junior Research Fellowship. P. P. Gopmandal kindly acknowledges the financial support by Science and Engineering Research Board, Govt. of India, through the project grant (File no. MTR/2018/001021).

Conflict of Interest The authors have declared no conflict of interest.

\section{References}

[1] Henry, D. C., Proc. R. Soc. London Ser. A 1931, 133, 106-129.

[2] Ohshima, H., Healy, T. W., White, L. R., J. Chem. Soc. Faraday Trans. 2 1983, 79, 16131628.

[3] Buitenhuis, J., Langmuir 2012, 28, 13354-13363.

[4] Bos, R., van der Mei, H. C., Busscher, H. J., Biophys. Chem. 1998, 74, 251-255.

[5] Duval, J. F. L., Gaboriaud, F., Curr. Opin. Colloid Interface Sci. 2010, 15, 184-195.

[6] Sonohara, R., Muramatsu, N., Ohshima, H., Kondo, T., Biophys. Chem. 1995, 5, 273-277.

[7] Daniel, M. C., Astruc, D., Chem. Rev. 2004, 104, 293-346.

[8] Lopez-Viota, J., Mandal, S., Delgado, A. V., Toca-Herrera, J. L., Moller Marco, Zanuttin, F., Balestrino, M., Krol, S., J. Colloid Interface Sci. 2009, 332, 215-223. 
[9] Helfricht, N., Doblhofer, E., Duval, J. F. L., Scheibel, T., Papastavrou, G., J. Phys. Chem. C. 2016, 120, 18015-18027.

[10] Duval, J. F. L., Wilkinson, K. J., van Leeuwen, H. P., Buffle, J., Environ. Sci. Technol. 2005, 39, 6435-6445.

[11] Town, R. M., Duval, J. F. L., Van Leeuwen, H. P., Environ. Sci. Technol. 2018, 52, 1168211690 .

[12] Ohshima, H., J. Colloid Interface Sci. 1997, 185, 269-273.

[13] Hill, R. J., Saville, D. A., Russel, W. B., J. Colloid Interface Sci. 2003, 258, 56-74.

[14] Lopez-Garcia, J. J., Horno, J., Grosse, C., J. Colloid Interface Sci. 2003, 268, 371-379.

[15] Mason, T. G., Lin, M. Y., Phys. Rev. E 2005, 71, 040801.

[16] Fernandez-Nievez, A., de las Nieves, F. J., Fernandez-Barbero, A., J. Chem. Phys. 2004, 120, 374-378.

[17] Duval, J. F. L., Ohshima, H., Langmuir 2006, 22, 3533-3546.

[18] Langlet, J., Gaboriaud, F., Gantzer, C., Duval, J.F.L., Biophys. J. 2008, 94, 3293-3312.

[19] Moussa, M., Caillet, C., Town, R. M., Duval, J. F. L., Langmuir 2015, 31, 5656-5666.

[20] Duval, J. F. L., Werner, C., Zimmermann, R., Curr. Opin. Colloid Interface Sci. 2016, 24, 112.

[21] Ohshima, H.,Kondo, T., Colloid Polym. Sci.1986, 264, 1080-1084.

[22] Ohshima, H., Kondo, T., J. Colloid and Interface Sci. 1987, 116, 305-311.

[23] Ohshima, H., Kondo, T., J. Colloid and Interface Sci. 1989, 130, 281-282.

[24] Ohshima, H., Kondo, T., Biophys. Chem. 1991, 39, 191-198.

[25] Ohshima, H., J. Colloid Interface Sci. 1994, 163, 474-483.

[26] Ohshima, H., Adv. Colloid Interface Sci. 1995, 62,189-235.

[27] Ohshima, H., J. Colloid Interface Sci. 2000, 228,190-193.

[28] Ohshima, H., J. Colloid Interface Sci. 2002, 252, 119-125.

[29] Ohshima, H., Electrophoresis 2006, 27, 526-533.

[30] Ohshima, H., Colloid and Surface A: Physicochem. Eng. Asp. 2011, 376, 72-75.

[31] Dingari, N. N., Buie, C. R., Langmuir 2014, 30, 4375-4384.

[32] Barbati, A. C., Kirby, B. J., Soft Matter2012, 8, 10598-10613.

[33] Andrews, J., Das, S., RSC Adv. 2015, 5, 46873-46880.

[34] Young, M. A., Jayaram, B., Beveridge, D. L., J. Phys. Chem. B1998, 102, 7666-7669.

[35] Lopez-Garcia, J. J., Horno, J., Grosse, C., J. Colloid Interface Sci. 2003, 268, 371-379.

[36] Ganjizade, A., Ashrafizadeh, S. N., Sadeghi, A., Electrochem. Commun. 2017, 84, 19-23.

[37] Poddar, A., Maity, D., Bandopadhyay, A., Chakraborty, S., Soft Matter 2016, 12, 5968-5978.

[38] Heyde, M. E., Peters, C. R., Anderson, J. E., J. Colloid Interface Sci. 1975, 50, 467-487.

[39] Coster, H. G. L., Biophys. J. 1973, 13, 133-142. 
[40] Maurya, S. K., Gopmandal, P. P., Bhattacharyya, S., Ohshima, H., Physical Review E 2018, 98, 023103.

[41] Ganjizade, A., Ashrafizadeh, S. N., Sadeghi, A., Colloid Polym. Sci. 2019, 297, 191-200.

[42] Maurya, S. K., Gopmandal, P. P., Ohshima, H., Duval, J. F. L., J. Colloid Interface Sci. 2020, 558, 280-290.

[43] Rochette, C. N., Crassous, J. J., Drechsler, M., Gaboriaud, F., Eloy, M., de Gaudemaris, B., Duval, J. F. L. Langmuir 2013, 29, 14655-14665.

[44] Duval, J. F. L., Farinha, J. P. S., Pinheiro, J. P. Langmuir 2013, 29, 13821-13835.

[45] Donath, E., Pastushenko, V., Bioelectrochem. Bioenerg. 1979, 6, 543-554.

[46] Wunderlich, R. W., J. Colloid Interface Sci. 1982, 88, 385-397.

[47] Israelachvili, J. N. Intermolecular and Surface Forces, Elsevier, 3rd ed, 2011, vol. 131, pp.7-8.

[48] Burgain, J., Scher, J., Lebeer, S., Vanderleyden, J., Corgneau, M., Guerin, J., Caillet, C., Duval, J. F. L., Francius, G., Gaiani, C., Colloids Surf. B: Biointerfaces 2015, 134, 332-338. [49] Von Smoluchowski, M., Z. Phys. Chem. 1917, 92, 129-168.

[50] Hyono, A., Gaboriaud, F., Mazda, T., Takata, Y., Ohshima, H., Duval, J. F. L. Langmuir 2009, 25, 10873-10885. 